\title{
NEAR INTEGRABLE SYSTEMS ON THE LINE. A CASE STUDY-PERTUBATION THEORY OF THE DEFOCUSING NONLINEAR SCHRÖDINGER EQUATION
}

\author{
Percy Deift ANd X. Zhou
}

In this letter we consider the Cauchy problem for perturbations

$$
i q_{t}+q_{x x}-2|q|^{2} q-2 \epsilon|q|^{l} q=0, \quad-\infty<x<\infty,
$$

of the integrable defocusing NLS equation

$$
i q_{t}+q_{x x}-2|q|^{2} q=0 .
$$

Here $\epsilon>0$ and $l>2$. We let $U_{t}^{\epsilon}\left(q_{0}\right)$ and $U_{t}^{\mathrm{NLS}}\left(q_{0}\right)$ denote the solutions at time $t$ of (1) and (2) respectively with initial data $q(x, t=0)=q_{0}(x)$. Of course, $U_{t}^{\mathrm{NLS}}\left(q_{0}\right)=U_{t}^{\epsilon=0}\left(q_{0}\right)$. For simplicity, we have chosen the perturbation in equation (1) to be proportional to $|q|^{l} q$. Our method extends directly, however, to perturbations of the form $\Lambda(|q|) q$, where $\Lambda(s)$ is a sufficiently smooth function behaving like $s^{l}$ near $s=0$.

In [McKSh], McKean and Shatah considered equations of the form

$$
i q_{t}+q_{x x}-2 \epsilon|q|^{l} q=0, \quad l>2,
$$

as perturbations of the free equation

$$
i q_{t}+q_{x x}=0
$$

Let $\tilde{U}_{t}\left(q_{0}\right)$ and $U_{t}^{F}\left(q_{0}\right)$ denote the solutions of (3) and (4) with initial data $q_{0}$ respectively. In [McKSh] the authors showed that for all initial data in the unit ball of a weighted Sobolev space, solutions of (3) with $\epsilon$ sufficiently small, behave asymptotically as $t \rightarrow \infty$ like solutions of the free equation (4), and the wave operator $\Omega_{+}$defined by

$$
\Omega_{+}\left(q_{0}\right) \equiv \lim _{t \rightarrow \infty} U_{-t}^{F} \circ \tilde{U}_{t}\left(q_{0}\right)
$$

Received May 30, 1997.

The first author was supported in part by NSF grant DMS-9500867, and the second author was supported in part by NSF grants DMS-9401403 and DMS-9304580. 
exists and is one-to-one onto a ball. Furthermore, $\Omega_{+}$conjugates the flows,

$$
U_{t}^{F} \circ \Omega_{+}=\Omega_{+} \circ \tilde{U}_{t}
$$

The result of McKean and Shatah provides a very interesting infinite dimensional example of when a given nonlinear equation $\dot{x}=f(x)$, with equilibrium point $x=0$, say, can be conjugated to its linearization $\dot{y}=f^{\prime}(0) y$ at the point. This subject has a large literature, devoted almost exclusively to the finite dimensional case: see for example, $[\mathrm{P}],[\mathrm{Si}],[\mathrm{H}],[\mathrm{Ni}]$, among many others.

The case $l=2$ (and $\epsilon=1$, which can of course always be achieved by scaling) in equation (3), corresponding to the integrable defocusing NLS equation, however, is critical: we see from [ZaMa],[DIZ], for example, that as $t \rightarrow \infty$ solutions $q(x, t)$ of NLS with Schwartz class initial data, have asymptotics

$$
q(x, t)=t^{-1 / 2} \alpha\left(z_{0}\right) e^{i x^{2} /(4 t)-i \nu\left(z_{0}\right) \log 8 t}+O\left(\frac{\log t}{t}\right),
$$

where $\alpha$ and $\nu$ are known functions of $z_{0}=x / 2 t$, and it follows easily that $\Omega_{+}$ in (5) does not exist. Nevertheless, an intertwining operator for the flows does exist.

To describe the intertwining operator, we recall the inverse scattering method for NLS [ZaSha]. Consider $2 \times 2$ matrix solutions $\psi=\psi(x, t ; z)$ of the Lax pair

$$
\begin{aligned}
\partial_{x} \psi & =P(x, t ; z) \psi, \\
\partial_{t} \psi & =V(x, t ; z) \psi,
\end{aligned}
$$

where

$$
\begin{aligned}
& P=i z \sigma+\left(\begin{array}{cc}
0 & q \\
\bar{q} & 0
\end{array}\right), \\
& V=-i z^{2} \sigma-z\left(\begin{array}{cc}
0 & q \\
\bar{q} & 0
\end{array}\right)+\left(\begin{array}{cc}
-i|q|^{2} & i q_{x} \\
-i \bar{q}_{x} & i|q|^{2}
\end{array}\right),
\end{aligned}
$$

and $\sigma=\left(\begin{array}{cc}\frac{1}{2} & 0 \\ 0 & -\frac{1}{2}\end{array}\right)$. The NLS equation (2) is equivalent to the commutation relation

$$
\left[\partial_{x}-P, \partial_{t}-V\right]=0
$$

The scattering map $\mathcal{R}$, is a map from potentials $q$ which decay sufficiently fast on $\mathbb{R}$, to reflection coefficients $\mathbf{r}$, and is defined as follows [ZaSha],[BC]. Given $q=q(x)$ and $\operatorname{Im} z \neq 0$, consider solutions $\psi=\psi(x, z)$ of (8) with the property

$$
\psi e^{-i x z \sigma} \text { approaches } I \text { as } x \rightarrow \infty \text { and is bounded as } x \rightarrow-\infty \text {. }
$$


For all $x$ and for all $z \in \mathbb{R}$, such solutions have boundary values $\psi_{ \pm}(x, z)=$ $\psi(x, z \pm i 0)$ from above and below, and satisfy the jump relations

$$
\psi_{+}(x, z)=\psi_{-}(x, z)\left(\begin{array}{cc}
1-|\mathbf{r}|^{2} & \mathbf{r} \\
-\overline{\mathbf{r}} & 1
\end{array}\right), \quad z \in \mathbb{R}
$$

where $\mathbf{r}=\mathbf{r}(z)$, the reflection coefficient for (8), is independent of $x$. We set

$$
\mathcal{R}(q)=\mathbf{r} .
$$

The map $\mathcal{R}$ linearizes the NLS equation (2) in the following way [ZaSha]: if $q(t)=q(x, t)$ is the solution of $(2)$ with initial data $q_{0}=q_{0}(x)=q(x, 0)$, then

$$
\mathcal{R}(q(t))=e^{-i t z^{2}} \mathcal{R}\left(q_{0}\right),
$$

or in terms of the reflection coefficient $\mathbf{r}(t)=\mathcal{R}(q(t))$,

$$
\mathbf{r}(t, z)=e^{-i t z^{2}} \mathbf{r}(0, z) .
$$

In terms of the flows $U_{t}^{\mathrm{NLS}}$ and $U_{t}^{F},(16)(17)$ imply

$$
T \circ U_{t}^{\mathrm{NLS}}=U_{t}^{F} \circ T
$$

where the intertwining operator $T$ is defined by

$$
T=\mathcal{F}^{-1} \circ \mathcal{R}
$$

and $\mathcal{F}$ in turn is the Fourier transform,

$$
(\mathcal{F} f)(z)=\int_{\mathbb{R}} e^{-i x z} f(x) d x .
$$

Remark 1 . The (inverse of the) intertwining operator $T$ is closely related to the so-called Dollard modified wave operator for the hydrogen Schrödinger equation,

$$
i \phi_{t}-H \phi=i \phi_{t}+\Delta \phi+\frac{\lambda}{|x|} \phi=0
$$

(see e.g. [RSi, Vol III]). In this case the wave operator s- $\lim _{t \rightarrow \infty} e^{i(-\Delta-\lambda /|x|) t} e^{i \Delta t}$ does not exist, but the modified wave operator $\Omega_{D}=\mathrm{s}-\lim _{t \rightarrow \infty} e^{i(-\Delta-\lambda /|x|) t}$ $e^{i\left(\Delta t+\frac{\lambda}{2 \sqrt{-\Delta}} \log t\right)}$ does exist. Hence, in one dimension, using the stationary phase method, we see that solutions $e^{-i H t} \phi$ of (21) evolves asymptotically as

$$
\begin{aligned}
e^{-i H t} \phi & \rightarrow e^{i(-\Delta-\lambda /|x|) t} e^{i\left(\Delta t+\frac{\lambda}{2 \sqrt{-\Delta}} \log t\right)}\left(\Omega_{D} \phi\right) \\
& \sim \operatorname{const.}\left(\mathcal{F}\left(\Omega_{D} \phi\right)\right)\left(z_{0}\right) e^{i z_{0}^{2} t+\frac{i \lambda}{2 z_{0}} \log t},
\end{aligned}
$$


as $t \rightarrow \infty$, where $z_{0}$ is again the point of stationary phase, $z_{0}=\frac{x}{2 t}$. On the other hand, inserting (7) into (2) we find that as $t \rightarrow \infty$

$$
i q_{t}+q_{x x}-\frac{2\left|\alpha\left(z_{0}\right)\right|^{2}}{t} q \sim 0 .
$$

But from [ZaMa] (see also [DIZ]) $\left|\alpha\left(z_{0}\right)\right|^{2}=\nu\left(z_{0}\right) / 2$, and hence

$$
i q_{t}+q_{x x}-\frac{2 z_{0} \nu\left(z_{0}\right)}{x} q \sim 0 .
$$

Comparing (24) with (21), we take $\lambda=-2 z_{0} \nu\left(z_{0}\right)$ and insert this value into (22), to obtain the asymptotic formula

$$
e^{-i H t} \phi \sim \text { const. } \frac{e^{i z_{0}^{2} t-i \nu\left(z_{0}\right) \log t}}{\sqrt{t}}\left(\mathcal{F}\left(\Omega_{D} \phi\right)\right)\left(z_{0}\right),
$$

which agrees exactly to leading order with (7). Thus solutions of NLS behave asymptotically like the modified "free" dynamics $e^{i z_{0}^{2} t+\frac{i \lambda}{2 z_{0}} \log t}$ in the Dollard wave operator.

In this letter we are concerned, in particular, with the following problem: Does an operator exist which intertwines the flows of the full equation (1) and its linearization (4), as in (18) above?

In the light of the preceding discussion, we cannot expect that the solutions of (1) behave asymptotically like solutions of the free equation (4). Rather, we expect that solutions of (1) behave like solutions of the integrable NLS equation (2). This in turn should give rise to an intertwining operator $\Omega_{+}^{\epsilon}$ for the flows $U_{t}^{\epsilon}$ and $U_{t}^{\mathrm{NLS}}$,

$$
\begin{gathered}
\Omega_{+}^{\epsilon}(q)=\lim _{t \rightarrow \infty} U_{-t}^{\mathrm{NLS}} \circ U_{t}^{\epsilon}(q), \\
U_{t}^{\mathrm{NLS}} \circ \Omega_{+}^{\epsilon}=\Omega_{+}^{\epsilon} \circ U_{t}^{\epsilon} .
\end{gathered}
$$

Then by (18),

$$
T^{\epsilon}=T \circ \Omega_{+}^{\epsilon}
$$

is an intertwining operator for $U_{t}^{\epsilon}$ and $U_{t}^{F}$,

$$
T^{\epsilon} \circ U_{t}^{\epsilon}=U_{t}^{F} \circ T^{\epsilon},
$$

as desired.

In order to state our results, we need some definitions. We denote by $|A|$ the Hilbert-Schmidt norm of the matrix $A=\left(A_{i j}\right),|A|=\left(\sum_{i, j}\left|A_{i j}\right|^{2}\right)^{1 / 2}$. For a matrix-valued function $f=f(x)$ on $\mathbb{R}$, define the weighted Sobolev space

$$
H^{k, j}=\left\{f: f, D^{k} f, x^{j} f \in L^{2}(\mathbb{R})\right\}, \quad k, j \geq 0
$$


with norm

$$
\|f\|_{H^{k, j}}=\left(\|f\|_{L^{2}}^{2}+\left\|x^{j} f\right\|_{L^{2}}^{2}+\left\|D^{k} f\right\|_{L^{2}}^{2}\right)^{1 / 2},
$$

where the $L^{2}$ norm of a matrix function $f$ is defined as the $L^{2}$ norm of $|f|$. For $\rho>0$, let

$$
H_{\rho}^{k, j}=\left\{f \in H^{k, j}:\|f\|_{L^{\infty}}<\rho\right\}, \quad k \geq 1, j \geq 0 .
$$

The map $q \mapsto r=\mathcal{R}(q)$ has many Fourier-like properties: for example, $\mathcal{R}$ takes the space of Schwartz class potentials $\{q\}$ onto the space of Schwartz class reflection coefficients $\{r\}$ with $\|r\|_{L^{\infty}}<1$ (see, for example, $[\mathrm{BC}]$ ). But more is true.

We say that a map $F$ from a subset $D$ of a Banach space $\mathcal{B}$ into $\mathcal{B}$ is (locally) Lipschitz if for each $q \in \mathcal{B}$, there is a neighborhood $N$ of $q$ and a positive number $L=L(N)$ such that

$$
\left\|F\left(q_{1}\right)-F\left(q_{2}\right)\right\|_{\mathcal{B}} \leq L(N)\left\|q_{1}-q_{2}\right\|_{\mathcal{B}}, \text { for all } q_{1}, q_{2} \in N \cap D .
$$

Proposition 1 [Z1]. The map $\mathcal{R}$ is bi-Lipschitz from $H^{k, j}$ onto $H_{1}^{j, k}$ for $k \geq 0$, $j \geq 1$.

In this letter, we consider (weak) solutions of equations (1) and (2) with initial data $q_{0} \in H^{1,1}$. Solutions in higher $H^{k, j}$ spaces can be analyzed in a similar manner (see Remark 5 below). Standard methods in PDE theory guarantee that if $q_{0}$ is in $H^{1,1}$, then the solutions $q(t)$ of (1) and (2) exist for all time in $H^{1,1}$ and are unique. Moreover, for any $t>0$ and $\epsilon \geq 0$, the map $q_{0} \mapsto U_{t}^{\epsilon}\left(q_{0}\right)$ is bi-Lipschitz from $H^{1,1}$ onto $H^{1,1}$.

Define

$$
G^{\epsilon}=\left\{q \in H^{1,1}: \Omega_{+}^{\epsilon}(q)=\lim _{t \rightarrow \infty} U_{-t}^{\mathrm{NLS}} \circ U_{t}^{\epsilon}(q) \text { exists in } H^{1,1}\right\}
$$

and for $\eta>0,0<\rho<1$,

$$
B_{\eta, \rho}=\left\{q \in H^{1,1}:\|q\|_{H^{1,1}}<\eta,\|\mathcal{R}(q)\|_{L^{\infty}}<\rho\right\} .
$$

A simple argument shows that $U_{t}^{\epsilon}\left(G^{\epsilon}\right) \subset G^{\epsilon}$ and the intertwining relation (26) is true on $G^{\epsilon}$. For $1<p<\infty$, let

$$
\rho_{p}=\frac{2}{1+\|S\|_{L^{p}(\mathbb{R}) \rightarrow L^{p}(\mathbb{R})}}<1
$$

where $S$ is the Hilbert transform on $\mathbb{R}$. Note that $\rho_{p} \rightarrow 0$ as $p \rightarrow \infty$.

In all the theorems that follow we assume that $l \geq 4$ (see Remarks 2 and 4 below). Our first result concerns the existence of $\Omega_{+}^{\epsilon}$ and its relation to the long-time behavior of $U_{t}^{\epsilon}\left(q_{0}\right)$. 
Theorem 1. Supose $\eta>0$ and $0<\rho<\rho_{4}<1$. Then

(i) there exists $\epsilon_{0}=\epsilon_{0}(\eta, \rho)>0$ such that

$$
B_{\eta, \rho} \subset G^{\epsilon}
$$

for all $\epsilon<\epsilon_{0}$,

(ii) $\Omega_{+}^{\epsilon}$ is Lipschitz from $B_{\eta, \rho}$ into $H^{1,1}$, and

(iii) for all $q \in B_{\eta, \rho}$,

$$
\left\|U_{t}^{\mathrm{NLS}}\left(\Omega_{+}^{\epsilon}(q)\right)\right\|_{L^{\infty}(d x)}=O\left(\frac{1}{t^{1 / 2}}\right),
$$

and

$$
\left\|U_{t}^{\epsilon}(q)-U_{t}^{\mathrm{NLS}}\left(\Omega_{+}^{\epsilon}(q)\right)\right\|_{L^{\infty}(d x)}=O\left(\frac{1}{t^{1 / 2+\kappa}}\right),
$$

for some $\kappa>0$, as $t \rightarrow \infty$.

The result (iii) above shows that solutions of equation (1) with initial data in the ball $B_{\eta, \rho}$, behave asymptotically, in the $L^{\infty}$ norm, like solutions of NLS.

Our next result concerns the range and invertibility of $\Omega_{+}^{\epsilon}$.

Theorem 2. For any $\epsilon>0$, there exists a Lipschitz map $\left(\Omega_{+}^{\epsilon}\right)^{\sharp}$ from $\mathcal{R}^{-1}\left(H_{\rho_{4}}^{1,1}\right)=\left\{q \in H^{1,1}:\|\mathcal{R}(q)\|_{L^{\infty}}<\rho_{4}\right\}$ to $G^{\epsilon} \subset H^{1,1}$ with the following property,

$$
\Omega_{+}^{\epsilon} \circ\left(\Omega_{+}^{\epsilon}\right)^{\sharp}=\mathbf{1}_{H_{\rho_{4}}^{1,1}} .
$$

In addition,

$$
\left(\Omega_{+}^{\epsilon}\right)^{\sharp} \circ \Omega_{+}^{\epsilon}=\mathbf{1}_{\left(\mathcal{R} \circ \Omega_{+}^{\epsilon}\right)^{-1} H_{\rho_{4}}^{1,1}} .
$$

Note that for $\epsilon<\epsilon_{0}(\eta, \rho)$ as in Theorem 1, condition (29) implies that $\Omega_{+} B_{\eta, \rho}$ is a neighborhood of 0 in $H_{\rho_{4}}^{1,1}$.

Conditions (29), (30) mean that $\left(\Omega_{+}^{\epsilon}\right)^{\#} \circ \Omega_{+}^{\epsilon}$ is the identity on its natural domain i.e. if $q \in G^{\epsilon}$, and $\Omega_{+}^{\epsilon}(q) \in \operatorname{Dom}\left(\Omega_{+}^{\epsilon}\right)^{\#}=\mathcal{R}^{-1} H_{\rho_{4}}^{1,1}$, then $\left(\Omega_{+}^{\epsilon}\right)^{\#} \Omega_{+}^{\epsilon}(q)=$ $q$. With this understanding of domains, we simply write $\left(\Omega_{+}^{\epsilon}\right)^{\#}=\left(\Omega_{+}^{\epsilon}\right)^{-1}$.

An immediate consequence of $(17),(26)$ and Theorem 2 is the following result.

Corollary to Theorem 2. (Conjugation of the flows) Let $\epsilon<\epsilon_{0}(\eta, \rho)$ as in Theorem 1, then $B_{\eta, \rho} \subset\left(\mathcal{R} \circ \Omega_{+}^{\epsilon}\right)^{-1} H_{\rho_{4}}^{1,1}$, and for all $q \in B_{\eta, \rho}$, and for all $t$,

$$
\begin{gathered}
U_{t}^{\epsilon}(q)=\left(\left(\Omega_{+}^{\epsilon}\right)^{-1} \circ U_{t}^{\mathrm{NLS}} \circ \Omega_{+}^{\epsilon}\right)(q), \\
U_{t}^{\epsilon}(q)=\left(\left(T^{\epsilon}\right)^{-1} \circ U_{t}^{F} \circ T^{\epsilon}\right)(q),
\end{gathered}
$$

where $T^{\epsilon}=T \circ \Omega_{+}^{\epsilon}$ as above.

The wave operator $\Omega_{-}^{\epsilon} \equiv \mathrm{s}-\lim _{t \rightarrow-\infty} U_{-t}^{\mathrm{NLS}} \circ U_{t}^{\epsilon}$ has similar properties to $\Omega_{+}^{\epsilon}$. Our final result describes the scattering theory for equation (1). 
Theorem 3. Let $\epsilon<\epsilon_{0}(\eta, \rho)$ as in Theorem 1. Define the scattering operator

$$
\mathcal{S}^{\epsilon}=\Omega_{+}^{\epsilon} \circ\left(\Omega_{-}^{\epsilon}\right)^{-1}
$$

on $\Omega_{-}^{\epsilon}\left(B_{\eta, \rho}\right)$. For $q_{-} \in \Omega_{-}^{\epsilon}\left(B_{\eta, \rho}\right)$, set $q_{+}=\mathcal{S}^{\epsilon}\left(q_{-}\right)$and define $q=\left(\Omega_{-}^{\epsilon}\right)^{-1}\left(q_{-}\right)=$ $\left(\Omega_{+}^{\epsilon}\right)^{-1}\left(q_{+}\right)$. Then as $t \rightarrow-\infty$,

$$
\left\|U_{t}^{\epsilon}(q)-U_{t}^{\mathrm{NLS}}\left(q_{-}\right)\right\|_{L^{\infty}}=O\left(\frac{1}{|t|^{\frac{1}{2}+\kappa}}\right)
$$

and as $t \rightarrow+\infty$,

$$
\left\|U_{t}^{\epsilon}(q)-U_{t}^{\mathrm{NLS}}\left(q_{+}\right)\right\|_{L^{\infty}}=O\left(\frac{1}{t^{\frac{1}{2}+\kappa}}\right)
$$

for some $\kappa>0$.

From (34) and (35) we see that the solution of (1) behaves like $U_{t}^{\mathrm{NLS}}\left(q_{-}\right)$as $t \rightarrow-\infty$, and like $U_{t}^{\mathrm{NLS}}\left(q_{+}\right)$as $t \rightarrow+\infty$, where $q_{+}=\mathcal{S}\left(q_{-}\right)$.

The first step in our method is to rewrite equation (1) in terms of the the reflection coefficient $\mathbf{r}(t)=\mathcal{R}(q(t))$. We recall some facts from inverse scattering theory [BC]. Set

$$
v(z)=\left(\begin{array}{cc}
1-|\mathbf{r}(z)|^{2} & \mathbf{r}(z) \\
-\overline{\mathbf{r}}(z) & 1
\end{array}\right)
$$

and

$$
v_{x}(z)=e^{i x z \sigma} v(z) e^{-i x z \sigma}=\left(\begin{array}{cc}
1-|\mathbf{r}(z)|^{2} & \mathbf{r}(z) e^{i x z} \\
-\overline{\mathbf{r}}(z) e^{-i x z} & 1
\end{array}\right)
$$

For a fixed $x$, the matrix function $m(x, z) \equiv \psi(x, z) e^{-i x a \sigma}$ solves the following Riemann-Hilbert (RH) problem (cf. 14),

(38a) $m(x, \cdot)$ is analytic in $\mathbb{C} \backslash \mathbb{R}$ and continuous up to the boundary,

(38b) $m_{+}(x, z)=m_{-}(x, z) v_{x}(z), \quad z \in \mathbb{R}$,

(38c) $\quad m_{ \pm}(x, z) \in I+L^{2}(\mathbb{R}, d z)$.

This RH problem is intimately connected with the scattering map $\mathcal{R}$. As indicated above, if $q \in H^{k, j}, k \geq 0, j \geq 1$, then $m=\psi e^{-i x z \sigma}$ solves the RH problem (38abc) with $\mathbf{r}=\mathcal{R}(q), v=\left(\begin{array}{cc}1-|\mathbf{r}|^{2} & \mathbf{r} \\ -\overline{\mathbf{r}} & 1\end{array}\right)$. Conversely, given $\mathbf{r}$ in $H_{1}^{j, k}, k \geq 0, j \geq 1$, then the $\mathrm{RH}$ problem (38abc) has a unique solution $m(x, z)=m(x, z ; \mathbf{r})$ with the property that $\psi=m e^{i x z \sigma}$ solves the first order equation $\left(\partial_{x}-P\right) \psi=0, P=i z \sigma+\left(\begin{array}{cc}0 & q \\ \bar{q} & 0\end{array}\right)$, with a potential $q$ in $H^{k, j}$, for which $\mathcal{R}(q)$ is precisely the given function $\mathbf{r}$. 
A simple calculation shows that equation (1) is equivalent to the commutator relation (cf. (12))

$$
\left[\partial_{x}-U, \partial_{t}-V\right]=\epsilon G
$$

where

$$
G=G(q)=-2 i|q|^{l}\left(\begin{array}{cc}
0 & q \\
-\bar{q} & 0
\end{array}\right)
$$

A computation similar to the standard proof of (16) (cf. [ZaSha],[AKNS]) leads to the following equation for $\mathbf{r}(t)=\mathcal{R}((q(t))$, which plays a central role in our analysis:

$$
\begin{aligned}
& \frac{d}{d t} \mathbf{r}(t, z)= \\
& -i z^{2} \mathbf{r}(t, z)+\epsilon \int_{-\infty}^{\infty} e^{-i z y}\left(m_{-}^{-1}(y, z ; \mathbf{r}(t)) G(q(y, t)) m_{-}(y, z ; \mathbf{r}(t))\right)_{12} d y
\end{aligned}
$$

where $(\quad)_{12}$ denotes the $(1,2)$-entry of the matrix. As $q(t)=\mathcal{R}^{-1}(\mathbf{r}(t))$, (41) should be viewed as an equation for $\mathbf{r}(t)$, equivalent to (1).

The key feature of equation (41) is that the "slowly decaying" term $-2|q|^{2} q$ is now removed, and the scattering map $q \mapsto \mathbf{r}$ can be viewed as a transformation to normal form. Indeed, substituting the relations

$$
\mathbf{r}=\mathcal{R}(q) \sim \mathcal{F}(q)
$$

and

$$
m_{-}(y, z ; \mathbf{r}) \sim I
$$

which are true for $q$ (equivalently $\mathbf{r}$ ) small, and taking the Fourier transform, we obtain precisely equation (3).

Morally, the above argument is correct: unfortunately, however, even if $q(t=$ 0 ) is sufficiently small, $q(t)$ in general does not remain small in the appropriate norm for all $t$. This leads to considerable complications in the analysis, and prevents us, at the technical level, from extending our results all the way to any $l>2$. Our method relies on a variety of estimates for the integrable NLS equation (2). These estimates are obtained using the non-linear steepest descent method for oscillatory RH problems introduced by the authors in [DZ]. In [DZ],[DIZ] the data is taken to have sufficiently high decay, and leads to an explicit asymptotic formula for the solutions (cf. (7)). In the present work, however, we can only assume that the data lies in $H^{1,1}$, and the explicit asymptotic form of the solution, with precise error estimates, is not clear. 
Our method, however, does not rely on explicit asymptotic formulae. We prove instead the following smoothing estimate for solutions of the NLS equation $(2)$ :

$$
\left|q^{\mathrm{NLS}}(x, t)\right| \leq \frac{C\left(\|\mathbf{r}(t=0)\|_{H^{1,0}},\|\mathbf{r}(t=0)\|_{L^{\infty}}\right)}{\sqrt{|t|}}\|\mathbf{r}(t=0)\|_{H^{1,0}}
$$

where $\mathbf{r}(t=0)=\mathcal{R}(q(t=0))$, and $C(\cdot, \cdot)$ is a smooth function of its arguments. Related, but weaker, smoothing estimates for NLS were obtained in [Z2]. Also certain smoothing estimates for KdV have been obtained by Kappeler [Ka], using the Gelfand-Levitan-Marchenko equation. Equation (44) plays a crucial role in an extended set of iterations leading to estimates for solutions of (41). As opposed to the case in which the unperturbed problem is linear, the fact that equation (2) is nonlinear leads to many complications, and multiplies considerably the number of estimates needed for the proof of Theorems 1-3. A manuscript with full details is in preperation.

Remark 2. The restriction $\|\mathbf{r}\|_{L^{\infty}}=\|\mathcal{R}(q)\|_{L^{\infty}}<\rho_{4}$ in our theorems, is an artifice of our method and arises in the following way. At certain points in the argument we need $L^{4}(d z)$ estimates for $m_{-}(x, \cdot ; \mathbf{r})$ : to obtain such estimates for $m_{-}$from the RH problem (38abc) we impose the small norm condition $\|\mathbf{r}\|_{L^{\infty}}<$ $\rho_{4}$. We believe that this condition is not necessary and $L^{4}(d z)$ bounds for $m_{-}$ can be obtained without this condition via a more detailed long-time analysis of solutions of the NLS equation in $H^{1,1}$. We plan to undertake such a detailed analysis in a future publication.

Remark 3. If $l \geq 6$, the small norm condition $\|\mathbf{r}\|_{L^{4}}<\rho_{4}$ is not needed. This is because the nonlinear term in equation (41) now decays sufficiently rapidly so that only $L^{2}(d z)$ (and not $L^{4}(d z)$ ) estimates for $m_{-}$are needed. Such estimates, however, are true for all $\mathbf{r}$ in $H^{1,1}$ with $\|\mathbf{r}\|_{L^{\infty}}<1$, and hence for all $q$ in $H^{1,1}$.

Remark 4. In all our results we have assumed that $l \geq 4$. This assumption was made to simplify and shorten the presentation, and our results actually extend to all $l>3 \frac{1}{2}$. As $l \rightarrow 3 \frac{1}{2}$, however, our method requires that $\|\mathbf{r}\|_{L^{\infty}}<\rho_{p}$ for larger and larger values of $p$. Recalling that $\lim _{p \rightarrow \infty} \rho_{p}=0$, this means that as $l \rightarrow 3 \frac{1}{2}$, our method applies only to Cauchy data $q(t=0)$ for equation (1) with smaller and smaller values of $\|\mathcal{R}(q(t=0))\|_{L^{\infty}}$. As noted in Remark 2, however, we expect that the condition $\|\mathbf{r}\|_{L^{\infty}}<\rho_{p}$ is in fact unnecessary.

Remark 5. In this letter we have only considered solutions of (1) in $H^{1,1}$. For any $k$ and $j$, analogous results can be proved for initial data in $H^{k, j}$, provided that (i) $k \geq j \geq 1$, and (ii) either $l$ is an even integer, or $l$ is sufficiently large to ensure the smoothness of (1).

In future publications we plan to extend the methods of this paper to analyze perturbations of a variety of integrable systems, including systems with soliton solutions. 
The theory of perturbations of integrable systems has generated a vast literature, and we conclude with a brief survey of results which are closest to ours. We will focus, in particular, on problems in $1+1$ dimensions.

Equations of the form (41) for a variety of systems of type

$$
q_{t}+K_{0}\left(q, q_{x}, q_{x x}, \cdots\right)=\epsilon K_{1}\left(q, q_{x}, \cdots\right)
$$

where $q_{t}+K_{0}\left(q, q_{x}, q_{x x}, \cdots\right)=0$ is integrable, were first derived in [K1], [KN] and [KarMas]. In these papers the authors used the equations of form (41), expanded formally in powers of $\epsilon$, to obtain information on solutions (in particular, soliton-type solutions) of (45) for times of order $\epsilon^{-\alpha}$ for some $\alpha>0$. Recently, Kivshar et al. [KiGSaV], and also Kaup [K2], have extended the method in $[\mathrm{K} 1],[\mathrm{KN}],[\mathrm{KarMas}]$ to obtain information for times of order $\epsilon^{-\alpha}$ for larger values of $\alpha$.

Results similar to those of $[\mathrm{K} 1],[\mathrm{KN}]$ and [KarMas] have been obtained by many authors, dating back to $[\mathrm{A}][\mathrm{McSc}],[\mathrm{W}]$, using the multi-scale/averaging method directly on the perturbed equation (45) (for further information, see $[\mathrm{AS}])$. We also refer the reader to an interesting recent preprint $[\mathrm{Br}]$ in which the author obtains similar results to those of Kivshar et al., using standard perturbation methods.

As we have noted in the body of the text, the map $q \mapsto \mathbf{r}=\mathcal{R}(q)$ can be viewed as a transformation of equation (1) to normal form. Kodama was the first to apply normal form ideas to nearly integrable $1+1$ dimensional systems, and in [Ko] he reduced (45), in the case of $\mathrm{KdV}$, to normal form up to order $\epsilon^{2}$. Kodama's transformation has been generalized recently by Fokas and Liu [FL].

In the spatially periodic case, rigorous normal form theorems have been obtained using infinite-dimensional KAM methods in the seminal work of Craig and Wayne $[\mathrm{CrW}]$ and also Kuksin $[\mathrm{Ku} 1],[\mathrm{Ku} 2]$. A consequence of this work is the proof of the existence of spatially and temporally periodic solutions of various evolution equations.

In a different direction, Ozawa in [O] considered solutions of generalized NLS equations

$$
i q_{t}+q_{x x}-\lambda|q|^{2} q-\mu|q|^{p-1} q=0, \quad-\infty<x<\infty,
$$

where $\lambda \in \mathbb{R} \backslash\{0\}, \mu \in \mathbb{R}$ and $p>3$. Under certain additional technical restrictions (e.g. $\mu \geq 0$ if $p \geq 5$ ), Ozawa used PDE methods to prove that modified Dollard-type wave operators $\Omega_{ \pm}^{\lambda, \mu}$ for (46) exist on a dense subset of a neighborhood of zero in $L^{2}(\mathbb{R})$ or $H^{1,0}(\mathbb{R})$. This means (cf. Remark 1 above) that solutions of (46) with initial data in $\operatorname{Ran} \Omega_{ \pm}^{\lambda, \mu}$ behave as $t \rightarrow \infty$ like solutions of the NLS equation

$$
i u_{t}+u_{x} x-\lambda|u|^{2} u=0 .
$$

In the case $\lambda>0$, these results are clearly related to Theorem 2 above. 
Finally we mention the fundamental work of Zakharov on normal form theory for nonlinear wave systems [Za]. A particularly illuminating exposition of the consequences of Zakharov's theory in the context of a class of $1+1$ dimensional dispersive wave equations, can be found in the recent paper of Majda et al. [MMcT].

\section{Acknowledgements}

The authors are especially grateful to Jalal Shatah for bringing the normal form problem for equation (1) to our attention and for many extremely helpful discussions. The authors would also like to thank Henry McKean, David McLaughlin, and Randall Pyke for providing us with additional useful information.

\section{References}

[A] M. J. Ablowitz, Applications of slowly varying nonlinear dispersive wave theories, Stud. Appl. Math. 50 (1971), 329-344.

[AKNS] M. J. Ablowitz, D. J. Kaup, A. C. Newell, and H. Segur, Method for solving the sine-Gordon equation, Stud. Appl. Math. 74 (1974), 249-315.

[AS] M. J. Ablowitz and H. Segur, Solitons and the inverse scattering transform, SIAM Stud. Appl. Math., SIAM, vol. 4, Philadelphia, 1981.

[BC] B. Beals and R. Coifman, Scattering and inverse scattering for first order systems, Comm. Pure Appl. Math. 37 (1984), 39-90.

[Br] J. C. Bronski, Nonlinear scattering and analyticity properties of solitons, preprint (1996).

[CrW] W. Craig and C. E. Wayne, Newton's method and periodic solutions of nonlinear wave equations, Comm. Pure Appl. Math. 46 (1993), 1409-1501.

[DIZ] P. Deift, A. Its and X. Zhou, Long-time asymptotics for integrable nonlinear wave equations, Important Developments in Soliton Theory 1980-1990, Springer-Verlag, 1993, pp. 181-204.

[DZ] P. Deift and X. Zhou, A steepest descent method for oscillatory Riemann-Hilbert problems-asymptotics for the MKdV equation, Ann. of Math. 137 (1993), 295-368.

[FL] A. S. Fokas and Q. M. Liu, Asymptotic integrability of water waves, Phys. Rev. Lett. 77 (1996), 2347-2351.

[H] P. Hartman, Ordinary differential equations (1964), J. Wiley \& Sons, New York.

[Ka] T. Kappeler, Solutions to the Korteweg-deVries equation with irregular initial profile, Comm. Part. Diff. Eq. 11 (1986), 927-945.

[KarMas] V. I. Karpman and E. M. Maslov, Structure of tails produces under the action of perturbations on solitons, Sov. Phys. JETP 48 (1978), 252-259.

[K1] D. J. Kaup, A perturbation expansion for the Zakharov-Shabat inverse scattering transform, SIAM Jour. Appl. Math. 31 (1976), 121-133.

[K2] $\quad$ Second-order perturbations for solitons in optical fibers, Phys. Rev. A 44 (1991), 4582.

[KN] D. J. Kaup and A. C. Newell, Solitons as particles, oscillators, and in slowly changing media: a singular perturbation theory, Proc. R. Soc. Lond. A. 361 (1978), 413-446.

[KigSaV] Y. S. Kivshar, S. A. Gredeskul, A. Sanchez and L. Vazques, Localization decay induced by strong nonlinearity in disordered systems, Phys. Rev. Lett. L 64 (1990), 1693. 
[Ko] Y. Kodama, On integrable systems with higher order corrections, Phys. Lett. A 107 (1985), 245-249.

[Ku1] S. Kuksin, Hamiltonian perturbations of infinite-dimensional linear systems with an imaginary spectrum, Funct. Anal. Appl. 21 (1987), 192-205.

[Ku2] _ Nearly integrable infinite-dimensional Hamiltonian systems, Springer Lecture Notes in Math., vol. 1556, Springer-Verlag, 1993.

[MMcT] A. J. Majda, D. W. McLaughlin and E. G. Tabak, A one-dimensional model for dispersive wave turbulence, J. Nonlinear Sci. 6 (1997), 9-44.

[MckSh] H. P. McKean and J. Shatah, The nonlinear Schrödinger equation and the nonlinear heat equation reduction to linear form, Comm. Pure Appl. Math. 44 (1991), 10671080.

[McSc] D. W. McLaughlin and A. C. Scott, Perturbation analysis of fluxon dynamics, Phys. Rev. A 18 (1978), 1652-1680.

[Ni] N. Y. Nikolenko, The method of Poincaré normal forms in problems of integrability of equations of evolution type, Russ. Math. Surveys 41 (1986), 63-114.

[O] T. Ozawa, Long range scattering for nonlinear Schrödinger equations in one space dimension, Comm. Math. Phys. 139 (1991), 479-493.

[P] H. Poincaré, Thèse, Oeuvres 1 (1928), Paris, 1879.

[RSi] M. Reed and B. Simon, Methods of modern mathematical physics. III. Scattering theory, Academic Press, New York-London, 1979.

[Si] C. L. Siegel, Über die Normalform analytischer Differentialgleichungen in the Nähe einer Gleichgewichtslösung, Akad. Wiss. Göttingen (1952), 21-30.

[W] G. B. Whitham, Linear and nonlinear waves, Pure and Applied Mathematics, Wiley-Interscience, New York-London-Sydney, 1974.

[Za] V. E. Zakharov, Kolmogorov spectra in weak turbulence problems, Handbook Plasma Phys. 2 (1984), 3-36.

[ZaMa] V. E. Zakharov and S. V. Manakov, Asymptotic behavior of nonlinear wave systems integrated by the inverse method, Sov. Phys. JETP 44 (1976), 106-112.

[KS] V. E. Zakharov and A. B. Shabat, Exact theory of two-dimensional self-focusing and one-dimensional self-modulation of waves in nonlinear media, Sov. Phys. JETP 34 (1972), 62-69.

[Z1] X. Zhou, The $L^{2}-$ Sobolev space bijectivity of scattering theory, Comm. Pure Appl. Math. (to appear).

[Z2] _ Strong regularizing effect of integrable systems, Comm. Part. Diff. Eq. 22 (1997), 503-526.

Courant Institute of Mathematical Sciences, New York University, New York, NY 10012-1110

E-mail address: deift@cims.nyu.edu

Department of Mathematics, Duke University, Durham, NC 27708-0320 and Institute for Advanced Study, Princeton, NJ 08540

E-mail address: zhou@math.duke.edu 